\title{
A Test for Equality of Exponential Distributions Based on Type-II Censored Samples
}

\author{
Kenneth N. Cole, Student Member IEEE \\ Air Force Institute of Technology, \\ Wright-Patterson AFB \\ Panna B. Nagarsenker \\ Air Force Institute of Technology, \\ Wright-Patterson AFB \\ Brahmanand N. Nagarsenker \\ Air Force Institute of Technology, \\ Wright-Patterson AFB
}

Key Words-Likelihood ratio test, beta distribution, percentage points.

\section{Reader Aids-}

Purpose: Widen state of the art

Special math needed for explanations: Complex analysis and statistics

Special math needed to use results: Statistics

Results useful to: Reliability theoreticians and statisticians

\begin{abstract}
Moments of the likelihood ratio test (LRT) criterion for testing the equality of several exponential distributions based on type-II censored samples of unequal size are obtained. They are used to obtain the distribution in a computational form for the first time. A table of selected significe points of LRT is presented.
\end{abstract}

\section{INTRODUCTION}

Most models for component \& system life \& reliability are based on the exponential distribution which plays much the same role in reliability theory that the $s$-normal distribution plays in sampling theory. Under some reasonably general conditions the distribution of the time between failures tends to an exponential as the complexity and the time of operation increase [2]. Epstein \& Sobel [5-7] and Epstein [3, 4] gave numerous results and popularized the exponential as a lifetime distribution, especially in the area of industrial life testing. This paper addresses the problem of testing the equality of several exponential distributions based on type-II censored samples of unequal size. The general moment of the likelihood ratio test (LRT) criterion is obtained and then this is used to obtain its distribution in a computational form. A short table of selected significance points of LRT is presented.

\section{PRELIMINARIES}

\section{Notation}

$p \quad$ number of samples

$i \quad$ sample serial number, $i=1,2, \ldots, p$ $n_{i} \quad$ number of observations in sample $i$

$r_{i} \quad$ number of failed items in sample $i$, predetermined prior to life testing, $1 \leqslant r_{i} \leqslant n_{i}$

$t_{i}(1) \leqslant t_{i(2)} \leqslant \ldots \leqslant t_{i\left(r_{i}\right)} \quad$ first $r_{i}$ observations in the sample $i$

$\Sigma_{i} \quad$ sum over $i$ from 1 to $p$

$\Pi_{i} \quad$ product over $i$ from 1 to $p$

$R \quad r_{1}+r_{2}+\ldots+r_{p}$; total number of failed items

$T_{i} \quad \sum_{j=1}^{r_{i}} t_{i(j)}+\left(n_{i}-r_{i}\right) t_{i\left(r_{i}\right)}, \quad$ total observed lifetime, or total time on test for sample $i$

$\lambda \quad$ likelihood ratio

LRT likelihood ratio test

$L \quad \lambda^{p / R}$

$H_{0} \quad$ null hypothesis

Other, standard notation is given in "Information for Readers \& Authors" at rear of each issue.

\section{Assumptions}

1. The $p s$-independent type II censored samples are available and sample $i$ has been drawn from an exponential distribution with pdf:

$\exp \left(t ; \theta_{i}\right)=\theta_{i}^{-1} \exp \left(-t / \theta_{i}\right), \quad$ for $t>0, \quad \theta_{i}>0 ;$

$$
\begin{aligned}
& i=1,2, \ldots, p \\
=0, & \text { otherwise }
\end{aligned}
$$

2. Each sample has unequal number of observations. The LRT for testing the hypothesis

$H_{0}: \theta_{1}=\theta_{2}=\ldots=\theta_{p}$

against the general alternatives, is:

$$
\begin{aligned}
& \lambda=\left(\Pi_{i} \hat{\theta}_{i}^{r_{i}}\right) / \hat{\theta}^{R} \\
& \hat{\theta}_{i}=T_{i} / r_{i} \\
& \hat{\theta}=\Sigma_{i} T_{i} / R
\end{aligned}
$$

The appendix shows that moment $h$ of $\lambda$ is:

$$
\mathrm{E}\left\{\lambda^{h}\right\}=K \cdot R^{R h} \cdot \Pi_{i}\left[r_{i}^{-h r_{i}} \Gamma\left\{r_{i}(1+h)\right\}\right] / \Gamma\{R(1+h)\}
$$

$$
K \equiv \Gamma(R) / \Pi_{i} \Gamma\left(r_{i}\right)
$$


It follows from (2.6) that moment $h$ of $L$ is:

$\mathrm{E}\left\{L^{h}\right\}=K \cdot R^{p h} \Pi_{i}\left[r_{i}^{-p h r_{i} / R} \Gamma\left\{r_{i}+p h r_{i} / R\right\}\right] / \Gamma(R+p h)$

\section{EXACT DISTRIBUTION OF $L$}

\section{Notation}

$\delta \quad$ convergence factor

pm $\quad R-\delta$

$k_{i} \quad r_{i} / R, \quad k_{1}+k_{2}+\ldots+k_{p}=1$

$B_{r}(\cdot) \quad$ Bernoulli polynomial of degree $r$ and order one $\operatorname{betf}(\cdot ; c, d) \quad$ beta Cdf, $\operatorname{betf}(x ; p, q) \equiv \int_{0}^{x} y^{p-1}(1-y)^{q-1}$ $d y / B(p, q)$, for $p>0, q>0$.

\section{Nomenclature}

Mellin transform The Mellin integral transform of a function $f(x)$, defined only for $x>0$ is:

$\mathrm{M}\{f(x) \mid s\}=\mathrm{E}\left\{x^{s-1}\right\}=\int_{0}^{\infty} x^{s-1} f(x) d x$ where $s$ is any complex variable.

$O(t) \quad f(t)$ is $O(t)$ if the function $f(t)$ is bounded by some constant multiple of $t$ for large $t$.

Apply the inverse Mellin transform [9] to the moment function of $L$ in (2.8); the $\operatorname{pdf}\{L\}$ is:

$$
\begin{aligned}
f(\ell)= & K \cdot(2 \pi i)^{-1} \int_{-i \infty}^{i \infty} \ell^{-h-1} \Pi_{i}\left[\left(k_{i}\right)^{-p h k_{i}} \Gamma\{(p m\right. \\
& \left.\left.+p h+\delta) k_{i}\right\}\right] \mathrm{dh} / \Gamma(p m+\delta+p h)
\end{aligned}
$$

Define

$t \equiv m+h$

Equation (3.3) results from (3.1) and (3.2):

$$
\begin{aligned}
& f(\ell)=K_{1} \cdot(2 \pi i)^{-1} \int_{m-i \infty}^{m+i \infty} \ell^{-t} \phi(t) d t \\
& K_{1} \equiv K \cdot \ell^{m-1} \Pi_{i}\left(k_{i}\right)^{p m k_{i}} \\
& \phi(t) \equiv \Pi_{i}\left[\left(k_{i}\right)^{-p t k_{i}} \Gamma\left\{(p t+\delta) k_{i}\right\}\right] / \Gamma(p t+\delta)
\end{aligned}
$$

Use the asymptotic expansion for log gamma function [1, pp 204]. Then

$$
\begin{aligned}
& \phi(t) \equiv K_{2} \cdot t^{-v}\left[1+q_{1} / t+q_{2} / t^{2}+\ldots\right] \\
& K_{2} \equiv(2 \pi / p)^{v} \Pi_{i}\left(k_{i}\right)^{\delta k_{i}-1 / 2} \\
& V \equiv(p-1) / 2
\end{aligned}
$$

The coefficients $q_{r}$ are recursively determined using (3.9) and (3.10):

$$
\begin{aligned}
& q_{r} \equiv \sum_{k=1}^{r} k A_{k} q_{r-k} / r, \quad q_{0}=1 \\
& A_{r} \equiv(-1)^{r}\left[B_{r+1}(\delta)-\Sigma_{i}\left\{B_{r+1}\left(\delta k_{i}\right) /\left(k_{i}\right)^{r}\right\}\right] / r(r+1) p^{r}
\end{aligned}
$$

Equation (3.6) shows that:

$$
\phi(t) / K_{2}=O\left(t^{-v}\right)
$$

with real part of $t$ tending to infinity; therefore $\phi(t)$ has the following exact representation as a factorial series [8]:

$\phi(t)=K_{2} \cdot \sum_{k=0}^{\infty} R_{i}\{\Gamma(t+a) / \Gamma(t+a+v+k)\}, \quad R_{0}=1$

where $a$ is a convergence factor chosen such that $R_{1}=0$ and the coefficients $R_{i}$ are obtained using the following recurrence relations from [8]:

$$
\begin{aligned}
& \sum_{j=0}^{i} R_{i-j} d_{i-j j}=q_{i}(i=1,2, \ldots) \\
& d_{i r}=\sum_{k=1}^{r} k c_{i k} d_{i r-k} / r, \quad d_{i 0}=1 \\
& c_{i r}=(-1)^{r-1}\left[B_{r+1}(a)-B_{r+1}(a+v+i)\right] / r(r+1)
\end{aligned}
$$

Use (3.12) in (3.3); the $\operatorname{pdf}\{L\}$ is [8]:

$f(\ell)=K_{3} \cdot \sum_{i=0}^{\infty} R_{i} \ell^{m+a-1}(1-\ell)^{v+i-1} / \Gamma(v+i)$

$K_{3} \equiv(2 \pi / p)^{v} \Gamma(R) \Pi_{i}\left[k_{i}^{R k_{i}-1 / 2} / \Gamma\left(R k_{i}\right)\right]$.

In (3.10), choose $\delta$ such that $A_{1}=0$. Then -

$$
\delta\left(\Sigma_{i} k_{i}^{-1}-1\right) / 6(p-1) .
$$

In (3.12), choose $a$ such that $R_{1}=0$. Then -

$a=(3-p) / 4$.

From (3.16), the $\operatorname{Cdf}\{L\}$ is:

$F(\ell)=K_{3} \cdot \sum_{i=0}^{\infty} R_{i}^{\prime} \operatorname{betf}(\ell, m+a, v+i)$

$$
R_{i}^{\prime} \equiv R_{i}\{\Gamma(m+a) / \Gamma(m+a+v+i)\}
$$

\section{NUMERICAL COMPUTATION}

The $\operatorname{Cdf}\{L\}$ in (3.20) is used to obtain $s$-significance points of $L$ for $p=2(1) 4, \alpha=0.005,0.01,0.025,0.05,0.1$, and with equal number of failures in each sample. The computations were carried out on Cyber 74 using double precision arithmetic and the results, to four significant 
TABLE 1

Percentage Points of $L=\lambda^{p / R}$ when $p=2$

\begin{tabular}{|c|c|c|c|c|c|}
\hline$x$ & 0.1 & 0.05 & 0.025 & 0.01 & 0.005 \\
\hline 3 & 0.613751 & 0.500527 & 0.405047 & 0.303874 & 0.243356 \\
\hline 4 & & & 0.513780 & 0.115398 & 0.352607 \\
\hline 5 & 0.752728 & .668214 & 0.590433 & 0.498895 & 0.438088 \\
\hline 6 & 0.790694 & 0.716537 & 0.646769 & 0.562583 & 0.505173 \\
\hline 7 & 0.818608 & 0.752684 & 0.689725 & 0.612381 & 0.558654 \\
\hline 8 & 0.839978 & 0.780716 & 0.723487 & 0.652237 & 0.602059 \\
\hline 9 & 0.856858 & 0.803074 & 0.750687 & 0.684790 & 0.637889 \\
\hline 10 & 0.870525 & 0.821313 & 0.773052 & 0.711816 & 0.667915 \\
\hline 11 & 0.881 .814 & 0.836472 & 0.791757 & 0.731671 & 0.693414 \\
\hline 12 & 0.091295 & 0.849267 & 0.807629 & 0.751175 & 0.715323 \\
\hline 13 & 0.899370 & 0.860209 & 0.021261 & 0.771027 & 0.731310 \\
\hline 14 & 0.906329 & 0.869674 & 0.033096 & 0.705731 & 0.750997 \\
\hline 15 & 0.912390 & 0.877941 & 0.843466 & 0.798669 & 0.765703 \\
\hline 16 & 0.917714 & 0.885223 & 0.852626 & 0.810141 & 0.778780 \\
\hline 17 & 0.922129 & 0.891686 & 0.860775 & 0.020302 & 0.790483 \\
\hline 18 & 0.926633 & 0.897161 & 0.868072 & 0.829577 & 0.801015 \\
\hline 19 & 0.930405 & 0.902652 & 0.874614 & 0.837880 & 0.810543 \\
\hline 20 & 0.933808 & 0.907343 & 0.880593 & 0.845114 & 0.819204 \\
\hline 25 & 0.946814 & 0.925338 & 0.903500 & 0.874576 & 0.852867 \\
\hline 30 & 0.955550 & 0.937483 & 0.919039 & 0.894493 & 0.875981 \\
\hline 35 & 0.961821 & 0.916230 & 0.930269 & 0.908956 & 0.892827 \\
\hline 40 & 0.966542 & 0.952831 & 0.938765 & 0.919935 & 0.905647 \\
\hline 45 & 0.970224 & 0.957988 & 0.945416 & 0.928552 & 0.915730 \\
\hline 50 & 0.973176 & 0.962129 & 0.950764 & 0.935495 & 0.923868 \\
\hline 55 & 0.975595 & 0.965527 & 0.955158 & 0.911208 & 0.930572 \\
\hline 60 & $0: 977614$ & 0.968366 & 0.958832 & 0.945993 & 0.936192 \\
\hline 65 & 0.979325 & 0.970772 & 0.961949 & 0.950057 & 0.940971 \\
\hline 70 & 0.980793 & 0.972839 & 0.964638 & 0.953552 & 0.945084 \\
\hline 75 & 0.982066 & 0.974632 & 0.966954 & 0.956591 & 0.948661 \\
\hline 80 & 0.983181 & 0.976204 & 0.968994 & 0.959256 & 0.941800 \\
\hline 85 & 0.984165 & 0.977592 & 0.970796 & 0.961613 & 0.954578 \\
\hline 90 & 0.985040 & 0.978827 & 0.972400 & $0.96371:$ & 0.957051 \\
\hline 95 & 0.985824 & 0.979933 & 0.973837 & 0.965594 & 0.959273 \\
\hline 0 & 0.986530 & 0.980929 & 0.975132 & $0.96729 \mathrm{u}$ & 0.961271 \\
\hline
\end{tabular}

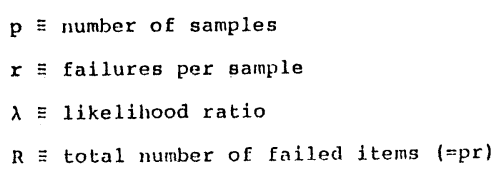

TABLE 2

Percentage Points of $L=\lambda^{p / R}$ when $p=3$

\begin{tabular}{|c|c|c|c|c|c|}
\hline$r$ & 0.1 & 0.05 & 0.025 & 0.01 & 0.005 \\
\hline 1 & 0.5418 & 0.1540 & 0.3704 & 0.2975 & 0.2181 \\
\hline 5 & 0.6183 & 0.5351 & 0.1631 & 0.3827 & 0.3313 \\
\hline $\begin{array}{l}6 \\
7\end{array}$ & $\begin{array}{l}0.6717 \\
0.7122\end{array}$ & 0.5959 & $\begin{array}{l}0.5288 \\
0.5807\end{array}$ & 0.4515 & 0.4007 \\
\hline & $\begin{array}{l}0.71222 \\
0.71339\end{array}$ & $\begin{array}{l}0.6131 \\
0.6806\end{array}$ & $\begin{array}{l}0.5807 \\
0.6223\end{array}$ & $\begin{array}{l}0.5075 \\
0.5536\end{array}$ & 0.4583 \\
\hline & 0.7694 & 0.7111 & 0.6571 & $\begin{array}{l}0.5536 \\
0.5921\end{array}$ & $\begin{array}{l}0.5065 \\
0.5472\end{array}$ \\
\hline 10 & 0.7902 & 0.7362 & 0.6859 & 0.6246 & 0.5819 \\
\hline & 0.8077 & 0.7574 & 0.7103 & 0.6525 & 0.6119 \\
\hline 12 & 0.8224 & 0.7755 & 0.7312 & 0.6765 & 0.6379 \\
\hline & 0.8351 & 0.7911 & 0.7493 & 0.6975 & 0.6607 \\
\hline $\begin{array}{l}14 \\
15\end{array}$ & $\begin{array}{l}0.8461 \\
0.8557 .\end{array}$ & $\begin{array}{l}0.8046 \\
0.8165\end{array}$ & $\begin{array}{l}0.7652 \\
0.7791\end{array}$ & $\begin{array}{l}0.7159 \\
0.7323\end{array}$ & $\begin{array}{l}0.6809 \\
0.6988\end{array}$ \\
\hline & 0.8642 & 0.8271 & 0.7916 & 0.7469 & 0.7148 \\
\hline 17 & 0.8718 & 0.8365 & 0.8027 & 0.7600 & 0.7293 \\
\hline & 0.8785 & 0.8449 & 0.8126 & 0.7718 & 0.7423 \\
\hline & 0.8846 & 0.8525 & 0.8217 & 0.7825 & 0.7542 \\
\hline & 0.8901 & 0.8594 & 0.8299 & 0.7923 & 0.7650 \\
\hline $\begin{array}{l}25 \\
30\end{array}$ & $\begin{array}{l}0.9113 \\
0.9256\end{array}$ & 0.8861 . & 0.8617 & 0.8304 & 0.8075 \\
\hline & $\begin{array}{l}0.9256 \\
0.9359\end{array}$ & $\begin{array}{l}0.9043 \\
0.9175\end{array}$ & $\begin{array}{l}0.8835 \\
0.8994\end{array}$ & $\begin{array}{l}0.8567 \\
0.8759\end{array}$ & $\begin{array}{l}0.8370 \\
0.8587\end{array}$ \\
\hline & 0.9437 & 0.9274 & $\begin{array}{l}0.8994 \\
0.9114\end{array}$ & 0.8907 & $\begin{array}{l}0.858 \\
0.8753\end{array}$ \\
\hline & 0.9499 & 0.9353 & 0.9209 & 0.9022 & 0.8884 \\
\hline & 0.9548 & 0.9416 & 0.9286 & 0.9116 & 0.8990 \\
\hline & 0.9588 & 0.9468 & 0.9349 & 0.9194 & 0.9078 \\
\hline & 0.9622 & 0.9511 & 0.9401 & 0.9258 & 0.915 .2 \\
\hline 65 & 0.9651 & 0.9548 & 0.9146 & 0.931 .4 & 0.9215 \\
\hline & 0.9675 & 0.9579 & $\begin{array}{r}0.9485 \\
0\end{array}$ & 0.9361 & 0.9269 \\
\hline & 0.9697 & 0.9607 & 0.9519 & 0.9403 & 0.9316 \\
\hline & $\begin{array}{l}0.9715 \\
0.9732\end{array}$ & 0.9631 & $\begin{array}{l}0.9548 \\
0.9574\end{array}$ & 0.9439 & $\begin{array}{l}0.9357 \\
0.9394\end{array}$ \\
\hline 90 & $\begin{array}{l}0.9742 \\
0\end{array}$ & 0.9672 . & $\begin{array}{l}0.9594 \\
0.9597\end{array}$ & $\begin{array}{l}0.9471 \\
0.9499\end{array}$ & 0.9427 \\
\hline 95 & 0.9759 & 0.9689 & 0.9618 & 0.9526 & 0.9456 \\
\hline & & & & & \\
\hline
\end{tabular}

figures, appear in tables 1-3. Percentage points of $L$ for the case of unequal number of failures are given in table 4 for $p=2$ and 3 and particular values of $k_{i}$.
TABLE 3

Percentage Points of $L=\lambda^{p / R}$ when $p=4$

\begin{tabular}{|c|c|c|c|c|c|}
\hline $\mathbf{r}$ & 0.1 & 0.05 & 0.025 & 0.01 & 0.005 \\
\hline 5 & 0.5215 & 0.4433 & $0.3780^{\prime}$ & 0.3072 & 0.2631 \\
\hline 6 & 0.5834 & 0.5099 & 0.4468 . & 0.3763 & 0.3309 . \\
\hline 7 & 0.6314 & 0.5629 & 0.5029 & 0.4343 & 0.3892 \\
\hline 8 & 0.6697 & 0.6059 & 0.5492 & 0.4833 & 0.4392 \\
\hline 9 & 0.7009 . & 0.6414 & 0.5879 & 0.5249 & 0.4822 \\
\hline 10 & 0.7268 & 0.6711 & 0.6206 & 0.5605 & 0.5194 \\
\hline 11 & 0.7486 & 0.6963 & 0.6486 & 0.5914 & 0.5519 \\
\hline 12 & 0.7672 & 0.7180 & 0.6728 & 0.6183 & 0.5804 \\
\hline 13 & 0.7832 & 0.7368 & 0.6910 & 0.6419 & 0.6056 \\
\hline 14 & 0.7972 & 0.7533 & 0.7126 & 0.6629 & 0.6279 \\
\hline 15 & 0.8095 & 0.7679 & 0.7291 & 0.6815 & 0.6480 \\
\hline 16 & 0.8204. & 0.7808 & 0.7438 & 0.6983 & 0.6661 \\
\hline 17 & 0.8302 & 0.7924 . & 0.7571 & 0.7134 & 0.6824 \\
\hline 18 & 0.8389. & 0.8028 & 0.7689 & 0.7271 & 0.6972 \\
\hline 19 & 0.8468 & 0.8123 & 0.7798 & 0.7395 & 0.7107 \\
\hline 20 & 0.8539 & 0.8209 & 0.7897 & 0.7508 & 0.7231 \\
\hline 25 & 0.8815 & 0.8542 & 0.8282 & 0.7955 & 0.7719 \\
\hline 30 & 0.9004 & 0.8771 & 0.8548 & 0.8266 & 0.8062 \\
\hline 35 & 0.9141 & 0.8938 & 0.8743 & 0.8495 & 0.8315. \\
\hline 40 & 0.9244 & 0.9065 & 0.8892 & 0.8671 & 0.8510 \\
\hline 45 & 0.9326 & 0.9165 & 0.9009 & 0.8811 & 0.8665 \\
\hline 50 & 0.9391 & 0.9245 & 0.9104 & 0.8923 & 0.8791 \\
\hline 55 & 0.9445 & 0.9312 & 0.9182 & 0.9016 & 0.8894 \\
\hline 60 & 0.9491 & 0.9367 & 0.9218. & 0.9095 & 0.8982 \\
\hline 65 & 0.9529 & 0.9415 & 0.9304 & 0.9162 & 0.9057 \\
\hline 70 & 0.9562 & 0.9155 & 0.9352 & 0.9219 & 0.9121 \\
\hline 75 & 0.9591 & 0.9491 & 0.9391 & 0.9269 & 0.9177 \\
\hline 80 & 0.9616 & 0.9522 & 0.9431 & 0.9314 & 0.9227 \\
\hline 85 & 0.9638 . & 0.9549 & 0.9463 & 0.9353 & 0.9271 \\
\hline 90 & 0.9658 & 0.9571 & 0.9493 & 0.9388 & 0.9310. \\
\hline 95 & 0.9676 & 0.9596 & 0.9519 & 0.9419 & 0.9345 \\
\hline 100 & 0.0601 & 0.9616 & 0.9512 & 0.9447 & 0.9377 \\
\hline
\end{tabular}

TABLE 4

Percentage Points of $L=\lambda^{p / R}, \alpha=.05$

\begin{tabular}{|c|c|c|c|c|c|c|}
\hline \multirow[b]{2}{*}{$k 1$} & \multirow[b]{2}{*}{$k 2$} & & \multicolumn{4}{|c|}{$\begin{array}{c}p=2 \\
R\end{array}$} \\
\hline & & & 10 & 20 & 60 & 100 \\
\hline 0.50 & 0.50 & & 0.66824365 & 0.82131333 & 0.93748256 & 0.96212923 \\
\hline 0.40 & 0.60 & & 0.66755956 & 0.82109744 & 0.93745503 & 0.96212238 \\
\hline 0.30 & 0.70 & & 0.66514658 & 0.82032925 & 0.93735617 & 0.96208578 \\
\hline 0.20 & 0.80 & & 0.65934591 & 0.81843068 & 0.93710946 & 0.96199434 \\
\hline \multirow[t]{2}{*}{0.10} & 0.90 & & 0.64306243 & 0.81251018 & 0.93630917 & 0.96169657 \\
\hline & & & \multicolumn{4}{|c|}{$\begin{array}{c}p=3 \\
R\end{array}$} \\
\hline$k 1$ & $k 2$ & $k 3$ & 10 & 20 & 60 & 100 \\
\hline 0.33 & 0.33 & 0.33 & 0.38374797 & 0.62865293 & 0.85946462 & 0.91350174 \\
\hline 0.30 & 0.30 & 0.40 & 0.38329741 & 0.62846305 & 0.85943505 & 0.91349039 \\
\hline 0.20 & 0.20 & 0.60 & 0.37670923 & 0.62564201 & 0.85899219 & 0.91332019 \\
\hline 0.20 & 0.30 & 0.50 & 0.38021160 & 0.62714428 & 0.85922828 & 0.91341034 \\
\hline 0.10 & 0.30 & 0.60 & 0.36943764 & 0.62211411 & 0.85840698 & 0.91309410 \\
\hline
\end{tabular}

\section{APPENDIX}

To obtain the moment $h$ of $\lambda$, we essentially use the method in [10] and the fact that under $H_{0}: \theta_{1}=\theta_{2}=\ldots=$ $\theta_{p}=\theta, T_{i}$ are $s$-independently distributed as gammas with parameters $r_{i}$ and $\theta$. For this consider the function $g(\mu)$ where:

$$
\begin{aligned}
g(\mu) & \equiv E\left[\Pi_{i}\left(T_{i}\right)^{h r_{i}} \exp \left\{\mu \Sigma_{i} T_{i}\right\}\right] \\
= & \theta^{R h} \cdot \Pi_{i} \frac{\Gamma\left\{r_{i}(1+h)\right\}}{\Gamma\left(r_{i}\right)}(1-\mu \theta)^{-(1+h) R} .
\end{aligned}
$$

The $\mathrm{E}\left\{\lambda^{h}\right\}$ is then obtained by taking derivative $r$ of $g(\mu)$ and then setting $r=-R h$ and $\mu=0$. This gives (2.6) of section 2 . 


\section{ACKNOWLEDGMENT}

An earlier version of this paper was presented at the 12th Annual Mini-Symposium of the American Institute of Aeronautics and Astronautics, held 1986 March 26 at the Air Force Institute of Technology, Wright-Patterson AFB.

\section{REFERENCES}

[1] T. W. Anderson, Introduction to Multivariate Statistical Analysis, John Wiley \& Sons, 1958.

[2] R. F. Drenick, "The failure law of complex equipment", Society for Industrial and Applied Mathematics, vol 8, 1960 Dec, pp 680-690.

[3] B. Epstein, "The exponential distribution and its role in life testing", Industrial Quality Control, vol 15, 1958 Dec, pp 5-9.

[4] B. Epstein, "Statistical life test acceptance procedures", Technometrics, vol 2, 1960 Nov, pp 435-446.

[5] B. Epstein, M. Sobel, "Life testing", J. Amer. Statist. Assoc., vol 48, 1953, pp 486-502.

[6] B. Epstein, M. Sobel, "Some theorems relevant to life testing from an exponential distribution", Ann. Math. Statist., vol 25, 1954, pp 373-381.

[7] B. Epstein, M. Sobel, "Sequential life tests in the exponential case", Ann. Math. Statist., vol 26, 1955, pp 82-93.

[8] B. N. Nagarsenker, P. B. Nagarsenker, "Distribution of LRT for testing the equality of several 2-parameter exponential distributions", IEEE Trans. Reliability, vol R-34, 1985 Apr, pp 65-68.

[9] E. C. Titchmarch, Introduction to the Theory of Fourier Integrals, Oxford University Press, 1948.

[10] S. S. Wilks, "Sample criteria for testing the equality of means, equality of variances and equality of covariances in a normal multivariate distribution", Ann. Math. Statist., vol 17, 1946, pp 257-281.

\section{AUTHORS}

Capt Kenneth N. Cole; Air Force Institute of Technology; WrightPatterson Air Force Base, Ohio 45433 USA.

Kenneth N. Cole [S'85] received BS in Business Management from the University of Maryland in 1967, BS in Electrical Engineering from the University of Florida in 1980 and MSEE from the Air Force Institute of Technology in 1983. From 1980 to 1982, Capt Cole was a Development Systems Engineer for the Air Force Aeronautical Systems Division at Wright-Patterson Air Force Base, Ohio. He is currently working towards his doctorate at the Air Force Institute of Technology. His research interests are in the areas of software systems supporting reliability studies and computer network environments. $\mathrm{He}$ is assigned to the Air Force Weapons Lab, Kirtland Air Force Base, New Mexico, beginning January 1987.

Panna B. Nagarsenker; Department of Mathematics and Computer Science; Air Force Institute of Technology; Wright-Patterson AFB, Ohio 45433, USA.

Panna B. Nagarsenker: For biography, see IEEE Trans. Reliability, vol R-34, 1985 Apr, p 68

Brahmanand N. Nagarsenker; Department of Mathematics and Computer Science; Air Force Institute of Technology; Wright-Patterson AFB, Ohio 45433, USA.

Brahmanand N. Nagarsenker: For biography, see IEEE Trans. Reliability, vol R-34, 1985 Apr, p 68.

Manuscript TR86-075 received 1986 June 19; revised 1986 November 4.

\section{Annual Reliability \& Maintainability Symposium Proceedings Price List for 1987}

Copies of past Proceedings are available (at the prices given below) at each Symposium or by mail from either of two places. Copies of recent proceedings are also available from the IEEE at prices set by the IEEE.

ASQC Bookmart

310 West Wisconsin Avenue

Milwaukee, Wisconsin 53203 USA

Ann. Reliability \& Maintainability Symp.

c/o Evans Associates

804 Vickers Avenue

Durham, North Carolina 27701 USA

Enclosing payment will speed delivery. Non-USA purchasers, please write for details about payment. Price includes postage by slow surface mail.
National Symposium on Reliability \& Quality Control (in Electronics)

First-Eleventh (1954-1965) . . . . . . . . not available

Reliability \& Maintainability Conference

First-Tenth (1962-1971) . . . . . . . . . . . not available

Annual Symposium on Reliability

1966-1971 ................... not available

Annual Reliability \& Maintainability Symposium

1972, 1979 . . . . . . . . . . . . . not available

$1973,1974,1975,1976,1977,1978, \ldots \ldots \ldots \$ 18$ each

$1980,1981,1982,1983 \ldots \ldots \ldots \ldots \ldots \ldots \ldots 24$ each

$1984,1985,1986,1987 \ldots \ldots \ldots \ldots \ldots \ldots \ldots$. . . . $\$ 40$.

1987 Tutorial Notes . . . . . . . . . . . . . . \$25 each 